\title{
Immediate postpartum levonorgestrel intrauterine device insertion and breast-feeding outcomes: a noninferiority randomized controlled trial
}

\author{
Dr David K. Turok, MD, MPH, Dr Lawrence Leeman, MD, MPH, Dr Jessica N. Sanders, PhD, \\ MSPH, Dr Lauren Thaxton, MD, MBA, Dr Jennifer L. Eggebroten, MD, Dr Nicole Yonke, MD, \\ MPH, Dr Holly Bullock, MD, MPH, Dr Rameet Singh, MD, MPH, Dr Lori M. Gawron, MD, MPH, \\ and Dr Eve Espey, MD, MPH \\ Department of Obstetrics and Gynecology, University of Utah, Salt Lake City, UT (Drs Turok, \\ Sanders, Eggebroten, Bullock, and Gawron), and Departments of Family and Community \\ Medicine (Drs Leeman and Yonke) and Obstetrics and Gynecology (Drs Leeman, Thaxton, Singh, \\ and Espey), University of New Mexico School of Medicine, Albuquerque, NM
}

\begin{abstract}
BACKGROUND-Immediate postpartum levonorgestrel intrauterine device insertion is increasing in frequency in the United States, but few studies have investigated the effect of early placement on breast-feeding outcomes.
\end{abstract}

OBJECTIVE-This study examined the effect of immediate vs delayed postpartum levonorgestrel intrauterine device insertion on breast-feeding outcomes.

STUDY DESIGN-We conducted this noninferiority randomized controlled trial at the University of Utah and the University of New Mexico Health Sciences Centers from February 2014 through March 2016. Eligible women were pregnant and planned to breast-feed, spoke English or Spanish, were aged 18-40 years, and desired a levonorgestrel intrauterine device. Enrolled women were randomized 1:1 to immediate postpartum insertion or delayed insertion at 4-12 weeks' postpartum. Prespecified exclusion criteria included delivery <37.0 weeks' gestational age, chorioamnionitis, postpartum hemorrhage, contraindications to levonorgestrel intrauterine device insertion, and medical complications of pregnancy that could affect breastfeeding. We conducted per-protocol analysis as the primary approach, as it is considered the standard for noninferiority studies; we also report the alternative intent-to-treat analysis. We powered the study for the primary outcome, breast-feeding continuation at 8 weeks, to detect a $15 \%$ noninferiority margin between groups, requiring 132 participants in each arm. The secondary study outcome, time to lactogenesis, used a validated measure, and was analyzed by survival

Corresponding author: David K. Turok, MD, MPH. David.Turok@hsc.utah.edu. Presented at the 2016 North American Forum on Family Planning, Denver, CO, Nov. 5-7, 2016.

The University of Utah Department of Obstetrics and Gynecology Program in Family Planning receives research funding from Bayer, Bioceptive, Contramed, Medicines 360, Merck, and Teva. D.K.T. serves on advisory boards for Allergan, Bayer, Pharmanest, and Teva. He is a consultant for Bioceptive and Contramed and a speaker for Allergan, Medicines 360, Merck, and Teva. The other authors have no conflicts of interest to report. 
analysis and log rank test. We followed up participants for ongoing data collection for 6 months. Only the data analysis team was blinded to the intervention.

RESULTS-We met the enrollment target with 319 participants, but lost 34 prior to randomization and excluded an additional 26 for medical complications prior to delivery. The final analytic sample included 132 in the immediate group and 127 in the delayed group. Report of any breast-feeding at 8 weeks in the immediate group (79\%; 95\% confidence interval, 70-86\%) was noninferior to that of the delayed group ( $84 \%$; 95\% confidence interval, 76-91\%). The 5\% difference in breast-feeding continuation at 8 weeks between the groups fell within the noninferiority margin ( $95 \%$ confidence interval, -5.6 to $15 \%$ ). Time to lactogenesis (mean \pm SD) in the immediate group, $65.3 \pm 25.7$ hours, was noninferior to that of the delayed group, 63.6 \pm 21.6 hours. The mean difference between groups was 1.7 hours ( $95 \%$ confidence interval, -4.8 to 8.2 hours), noninferior by log-rank test. A total of 24 intrauterine device expulsions occurred in the immediate group compared to 2 in the delayed group (19\% vs $2 \%, P<.001)$, consistent with the known higher expulsion rate with immediate vs delayed postpartum intrauterine device insertion. No intrauterine device perforations occurred in either group.

CONCLUSION-Our results of noninferior breast-feeding outcomes between women with immediate and delayed postpartum levonorgestrel intrauterine device insertion suggest that immediate postpartum intrauterine device insertion is an acceptable option for women planning to breast-feed and use the levonorgestrel intrauterine device. Expulsion rates are higher with immediate postpartum levonorgestrel intrauterine device insertion compared to delayed insertion, but this disadvantage may be outweighed by the advantages of immediate initiation of contraception. Providers should offer immediate postpartum intrauterine device insertion to breastfeeding women planning to use the levonorgestrel intrauterine device.

\section{Keywords}

breast-feeding; contraception; immediate postpartum intrauterine device insertion; levonorgestrel intrauterine device

\section{Introduction}

Breast-feeding provides health benefits to women and infants. ${ }^{1}$ The World Health Organization recommends exclusive breast-feeding for 6 months postpartum and continuation of breast-feeding through 2 years as a best practice for infant nutrition and maternal health. ${ }^{2}$ Although $80 \%$ of US women plan to breast-feed, ${ }^{3}$ only $18.8 \%$ exclusively breast-feed at 6 months, short of the Centers for Disease Control and Prevention (CDC) Healthy People 2020 objective of $25 \% .^{4}$

Postpartum women are frequently motivated to prevent pregnancy and may appreciate the convenience of initiating a highly effective contraceptive method in the hospital rather than waiting for a postpartum visit. ${ }^{5,6}$ Women receiving immediate postpartum intrauterine devices (IUDs) report high satisfaction, and continuation rates. ${ }^{7}$ When the levonorgestrel (LNG) IUD is placed immediately postpartum there is a significantly higher rate of expulsion compared to women initiating the IUD at the postpartum visit. ${ }^{8}$ Despite the higher expulsion with immediate postpartum placement, accounting for replacement of expelled 
IUDs, continuation rates at 6 and 12 months are similar to those in women initiating the IUD at the postpartum visit. ${ }^{7}$

The advantages of immediate postpartum IUD placement, including preventing unintended pregnancy, ${ }^{9}$ must be weighed against potential drawbacks. Rapid clearance of progesterone after placental delivery triggers lactogenesis, raising concerns that immediate postpartum initiation of the progestin-secreting LNG IUD could disrupt lactogenesis and reduce breast milk production, ${ }^{10}$ even though $\mathrm{LNG}$ is an androgen derivative and not a true progestin. Providers often discourage immediate initiation of hormonal contraception, including the LNG IUD, due to concerns of a negative impact on breast-feeding, ${ }^{11}$ but data on the effect of hormonal intrauterine contraception on breast-feeding are limited. ${ }^{12}$ Some studies assessing early exposure to oral contraception ${ }^{13}$ and in-hospital post-partum etonogestrel implant insertion ${ }^{14}$ have shown no adverse effect on lactation. These results prompted our use of a noninferiority design for the current study.

Based on minimal evidence, the CDC Medical Eligibility Criteria for Contraceptive Use assign a category 2 rating (the advantages generally outweigh the theoretical or proven risks) to immediate postpartum LNG IUD initiation in breast-feeding women. ${ }^{15}$ This study assesses the effect of immediate vs delayed postpartum initiation of LNG IUD on breastfeeding outcomes. The primary outcome was report of any breast-feeding at 8 weeks' postpartum.

\section{Materials and Methods}

\section{Trial design and oversight}

The Breastfeeding LNG IUD Study, a noninferiority randomized controlled trial, was conducted at the University of Utah and the University of New Mexico Health Sciences Centers from February 2014 through March 2016. Each site's institutional review board approved the study (Clinicaltrials.gov registry number NCT01990703).

\section{Study procedures}

Study staff approached and enrolled potential participants during prenatal care who expressed intent to breast-feed and desired a LNG IUD for postpartum contraception. Eligible women presenting for induction of labor or in early, but not active, labor were also approached on labor and delivery. Potential participants received standardized counseling about the risks and benefits of immediate postpartum vs delayed LNG IUD insertion, including increased risk of IUD expulsion and unknown effect of LNG IUD on breastfeeding with immediate postpartum insertion. After screening for eligibility and obtaining informed consent, a clinical research team member enrolled participants. Baseline data included age, gravidity, parity, contraceptive history, and breast-feeding experience.

Eligible participants included healthy pregnant women fluent in English or Spanish aged 1840 years, who desired the LNG IUD as their postpartum method of contraception, planned to breast-feed, and agreed to randomization of LNG IUD placement timing. Prespecified exclusion criteria included delivery <37.0 weeks' gestational age, clinical chorioamnionitis, postpartum hemorrhage, preeclampsia with severe features, prolonged antepartum 
hospitalization, coagulopathy, hepatic disease, undiagnosed genital bleeding, or relative contraindications to LNG IUD insertion.

Randomization with equal (1:1) probability of assignment to the immediate or delayed group occurred via computerized block randomization with alternating block sizes of 4,6 , and 8. The lead statistician (J.N.S.) generated treatment assignments for each institution that were stored in secure spreadsheet software files and uploaded into the Research Electronic Data Capture program, a secure Web-based application, ${ }^{16}$ which was also used for electronic data collection and data entry. Random assignment to one of the treatment groups occurred on the labor and delivery unit at the time of presentation for delivery. The provider contacted a site research coordinator to obtain assignment information. Participants and clinicians were not blinded to intervention assignment; the data analysis team remained blinded.

\section{Intervention}

Both sites conducted didactic training on immediate postpartum IUD insertion with modelbased simulation. At the New Mexico site providers completed a certification process with 3-5 insertions performed under direct supervision of a certified attending physician. The Utah site required supervision by an experienced attending physician until trainees were determined to be competent. New Mexico providers used standard 2-ring forceps insertion technique ( 1 on anterior cervical lip, 1 holding IUD); the Utah site encouraged insertions with Kelly placenta forceps to hold the IUD and permitted hand placement. These practice variations were not expected to affect study outcomes.

All immediate postpartum IUD insertions were performed by obstetrician-gynecology and family medicine physicians following delivery of the placenta with a goal of placement within 30 minutes. Although not required, physicians routinely used ultrasound guidance for insertions after vaginal delivery. Women in the immediate group returned at 2-4 weeks' postpartum to confirm IUD retention. Delayed insertions occurred at the participant's usual clinic by an experienced provider 4-12 weeks' postpartum.

Following enrollment, randomization, and delivery of a healthy term infant, the clinical research coordinators at each site contacted their respective site participants twice daily prior to hospital discharge. Time to lactogenesis was documented by maternal perception, as described and validated by Chapman and Perez-Escamilla ${ }^{17}$ and used in a prior study conducted at the Utah site. ${ }^{14}$ If lactogenesis had not occurred prior to hospital discharge, the participant received daily text messages or telephone calls from study personnel up to 5 days postpartum until lactogenesis was confirmed and recorded. Delayed lactation was defined as lack of production of breast milk by 120 hours post-partum as assessed by maternal perception. Lactation failure was defined as no breast milk production by 2 weeks' postpartum.

Study staff contacted participants by telephone, text messaging, or electronic communication weekly from 2-12 weeks' postpartum to collect data on breast-feeding continuation, type of breast-feeding (exclusive breast-feeding, breast-feeding and pumping, or breast-feeding with supplementation), and date of cessation, if applicable. Participants were evaluated in-person at the prearranged postpartum visit. There was a final follow-up at 6 months' post-partum. 
We also tracked contraceptive method use, including dates of expulsions and reinsertions if they occurred, method satisfaction, and adverse events including any hospitalizations or events related to IUD use.

Participants were not charged for IUDs or insertion procedures. Due to lack of Medicaid and private insurance reimbursement for in-hospital placement in Utah, the site only offered enrollment to women who qualified through an externally funded training grant for uninsured women. For women who had coverage in New Mexico, Medicaid or private insurance was billed for the insertion procedure. In the event of IUD expulsion or desired removal, clinic personnel counseled participants on options for reinsertion or contraceptive alternatives. We billed these visits to insurance and for uninsured women the study covered the charge.

\section{Sample size}

Based on a prior New Mexico study, we expected $60 \%$ of women with delayed initiation of hormonal contraception to continue breast-feeding at 8 weeks' postpartum. ${ }^{13}$ We chose any breast-feeding at 8 weeks' postpartum as the primary outcome measure since duration of breast-feeding is clinically meaningful. We assumed that if negative breast-feeding effects resulted from early exogenous hormonal exposure, they would occur early in lactation. For the primary outcome, we used a noninferiority margin of $15 \%$ for 8 -week breast-feeding continuation rates between the immediate group and the delayed group based on the research team's estimate of a clinically significant difference. To achieve $80 \%$ power with a 1 -sided type 1 error of $5 \%$ utilizing the $\mathrm{Z}$ test (unpooled), 132 participants were required in each group. To obtain 264 participants with complete data, we increased enrollment by $20 \%$ and aimed to enroll a total of 317 participants. For assessment of lactogenesis, we used a previously established clinically meaningful noninferiority margin of 8 hours between groups, requiring 34 participants per group. ${ }^{14}$ The sample size calculation was based on a mean lactogenesis time of 54 hours and SD of 12 hours. The study sample size far exceeded the sample necessary to test this outcome.

\section{Statistical analysis}

We summarized the demographic characteristics and the primary outcome of breast-feeding continuation and the secondary outcome of lactogenesis and assessed the differences between assigned groups using Pearson $\chi^{2}$ test or binomial 95\% confidence intervals (CI) for categorical variables and Wilcoxon rank sum test or Student $t$ test for continuous variables. The per-protocol analysis is presented as the primary approach throughout the article; it is considered the gold standard in noninferiority trials, as it is more conservative than the intent-to-treat approach. ${ }^{18,19} \mathrm{We}$ also report outcomes using intent-to-treat analysis.

Per-protocol analysis included women who received their treatment according to the prespecified protocol. For the immediate group, per-protocol analysis included participants who received immediate postpartum IUD insertion with any immediate exposure; this includes individuals with IUD expulsion prior to 8-week follow-up. For the delayed group, per-protocol analysis included only women with documented receipt of a delayed postpartum IUD (at 4-12 weeks). The intent-to-treat analysis included all women who were 
randomized and did not develop a medical complication leading to exclusion prior to delivery. In both the intent-to-treat and per-protocol analyses breast-feeding rates were compared using the 1 -sided $\chi^{2}$ test of noninferiority. If the CI did not cross the prespecified $15 \%$ margin, noninferiority was confirmed. ${ }^{19}$

The secondary outcome of lactogenesis was assessed using a survival analysis and log-rank test to compare the survival distributions timing of lactogenesis between groups, including individuals with delayed lactogenesis, defined as milk coming in >120 hours. Mean times to lactogenesis are reported excluding those individuals who had delayed lactogenesis or lactation failure.

We also conducted post hoc exploratory analyses of the treatment effect (immediate postpartum insertion vs delayed IUD insertion) on outcomes up to 6 months postpartum, including any or exclusive breast-feeding, IUD expulsion, and patient satisfaction with the timing of LNG IUD insertion. For satisfaction, we employed a 0 -to-100 scale assessing "how happy are you with the IUD received" (anchors of 0 "not happy at all" to 100 "very happy") assessed by Pearson $\chi^{2}$ test, or Fisher exact test as appropriate. In addition, we asked participants their perception of optimal timing for insertion of a contraceptive relative to a birth. Response options included "in the hospital at the time of birth," "4 weeks after the birth," "at a 6-week postpartum exam," or "at another clinic visit 6-8 weeks after birth." We used software (STATA 14; StataCorp LP, College Station, TX) for statistical analysis.

\section{Results}

\section{Participant characteristics}

From February 2014 through March 2016, we assessed 453 women for eligibility; 319 consented to study participation and enrolled. Enrollment ceased when the target number of participants had consented to participation. The details regarding the 34 (11\%) nonrandomized participants are included in Figure 1. Randomization assigned 147 women to the immediate and 138 women to the delayed group. The Table includes all randomized participants who were not excluded for medical complications prior to delivery. There were no differences between the 2 groups of baseline and demographic characteristics.

After randomization, 26 women experienced medical complications during labor and delivery and were excluded from both analytic approaches. In all, 22 women in the immediate group and 35 in the delayed group did not receive their allocated intervention. Postrandomization exclusion criteria for per-protocol analysis included protocol violations and participant withdrawals prior to hospital discharge. We retained data for women in the delayed insertion group who were lost to follow-up or withdrew from the study after primary outcome data were collected ${ }^{20}$ (Figure 1).

Per-protocol assessment included 228 women who completed the assigned treatment (125 in the immediate and 103 in the delayed group). A total of 259 participants were included in the intent-to-treat analysis (132 women in the immediate and 127 in the delayed group). 


\section{Primary outcome: 8-week breast-feeding continuation}

Per-protocol analysis demonstrated noninferiority between immediate postpartum LNG IUD insertion and delayed insertion for the primary outcome of any breast-feeding at 8 weeks: $79 \%$ (95\% CI, 70-86\%) vs 84\% (95\% CI, 76-91\%) $(P=.28)$ (Figure 2). Intent-to-treat analysis demonstrated similar findings: $78 \%$ (95\% CI, 70-85\%) and 83\% (95\% CI, 75-90\%) ( $P=.36$ ) of women in the 2 groups, respectively, who continued breast-feeding at 8 weeks. The 5\% difference in breast-feeding continuation at 8 weeks between the groups fell within the noninferiority margin ( $95 \% \mathrm{CI},-5.6$ to $15 \%)$. A Cox proportional hazard ratio model controlling for randomization, site, age, ethnicity, epidural, and parity showed no significant associations between these factors and the primary outcome measure.

\section{Secondary outcome: time to lactogenesis}

Survival distributions of time to lacto-genesis for both groups are illustrated in the KaplanMeier survival curve (Figure 3). The distributions of time to lactogenesis did not differ between groups as evaluated by the log-rank test $(P=.22)$ (Figure 3$)$. Mean times to lactogenesis, excluding women with delayed lactation, occurred at (mean \pm SD) $65.3 \pm 25.7$ hours for the immediate group and $63.6 \pm 21.7$ hours for the delayed group. The mean difference was 1.7 hours ( $95 \% \mathrm{CI},-4.8$ to 8.2 hours; $P=.61$ ). Delayed lactation occurred in 11 women $(9 \%)$ in the immediate group and $6(6 \%)$ in the delayed group $(P=.46)$. Two women experienced complete lactogenesis failure in the per-protocol approach, both in the immediate group.

\section{Post hoc exploratory outcomes}

For the exploratory outcome of exclusive breast-feeding at 8 weeks, defined as no breast milk pumping, formula supplementation, or use of solid foods, the per-protocol analysis found no significant differences between the immediate and delayed groups: 33\% (95\% CI, $24-43 \%)$ and $40 \%(95 \% \mathrm{CI}, 31-51 \%)(P=.27)$ (Figure 4$)$.

IUD expulsions were more common in the immediate postpartum (24/125 [19\%]) compared to the delayed (2/103 [2\%]) group. The majority of women in the immediate postpartum group (17/24 [71\%]) who experienced IUD expulsion requested and received IUD reinsertion at their postpartum clinic visit. Both groups reported high 3- and 6-month satisfaction rates. The average satisfaction score measured at 12 weeks' postpartum on a 0 to-100 scale among the immediate postpartum group was 86 (95\% CI, 80-91\%) and in the delayed group was 87 (95\% CI, 79-96\%). When participants completed the 12-week questionnaire, $80 \%$ in the immediate postpartum group and $42 \%$ in the delayed group responded that, "in the hospital at the time of birth is the perfect time to have a birth control device placed."

\section{Safety monitoring}

No uterine perforations or uterine infections were reported in this study either with immediate postpartum or delayed IUD insertion. 


\section{Comment}

This randomized controlled trial demonstrated that breast-feeding outcomes in women with immediate post-partum LNG IUD insertion were noninferior to those in women with delayed insertion. This study further supports prior literature showing no adverse effect of progestin-only contraceptives on lactation, ${ }^{20}$ and high levels of contraceptive satisfaction for women in both groups. ${ }^{7}$

The limited data evaluating potential harms of immediate postpartum initiation of progestinonly contraceptives on lactation show mixed results. A systematic review of progestin-only contraceptives demonstrated no adverse effects on breast-feeding or infant outcomes, ${ }^{21}$ while a secondary analysis of 59 breast-feeding women randomized to immediate postpartum vs delayed LNG IUD insertion found that fewer women in the immediate insertion group breast-fed at 6 months. ${ }^{12}$ A randomized trial of inhospital postpartum etonogestrel implant insertion compared with standard postpartum insertion found no differences in time to lactogenesis, formula supplementation, or milk composition, similar to the findings of the current study. ${ }^{14}$ A nonrandomized prospective trial compared women initiating progestin-only methods (mostly depo medroxyprogesterone acetate) within the first 3 postpartum days with women using nonhormonal methods and found no differences in breast-feeding outcomes. ${ }^{22}$

Major strengths of our study include its randomized controlled design, use of study sites that had incorporated the intervention into routine care prior to study initiation, and lack of industry sponsorship. Study endpoints of breast-feeding continuation and lacto-genesis are clinically relevant.

Study limitations include lack of ability to blind the timing of LNG IUD placement, loss to follow-up, lack of long-term infant and childhood outcomes, and limited applicability as we included only women having a term birth. Because we did not collect data on breast milk volume per feeding and the number and duration of breast-feeding sessions, we did not specifically address whether early postpartum exposure to a LNG IUD decreases milk production. ${ }^{23}$ Challenges to internal validity include a smaller final sample than intended despite meeting enrollment goals. The smaller sample size resulted from a greater than expected number of exclusions both after enrollment and post-randomization. An additional challenge to internal validity resulted from loss to follow-up of 12 of the 13 participants in the early insertion group who had IUD expulsions. The omission of these immediate insertion participants from the primary outcome could potentially widen the CI of the perprotocol analysis, resulting in a more conservative estimate of the noninferiority result. Our sample size was insufficient to power 2 important subgroup analyses. Nearly three quarters of participants had prior breast-feeding experience, although we were unable to assess differences in continuation based on prior breast-feeding. Our ability to assess lactation failure was limited given the rarity of the outcome in our sample. Another study limitation comes from a strength in our hospitals: both sites have Baby-Friendly Hospital Initiative accreditation. ${ }^{24}$ This supportive breast-feeding environment may have independently contributed to attainment of the CDC Healthy People 2020 objective of $25 \%$ of mothers exclusively breast-feeding at 6 months postpartum. ${ }^{4}$ 
Effective contraception reduces rapid repeat pregnancies and poor perinatal outcomes. ${ }^{25} \mathrm{In}$ one study, $42 \%$ of women were interested in initiating implants and IUDs immediately postpartum. ${ }^{6}$ However, many women do not return for postpartum follow-up appointments or become pregnant prior to the visit and only $81 \%$ of women randomized to delayed postpartum IUD insertion ultimately received the device, reflecting barriers inherent in deferring contraceptive initiation. ${ }^{26,27}$ Similarly, in this study 24 women assigned to delayed insertion did not return for IUD insertion and 9 participants received their IUD beyond the primary study outcome timeline of 8 weeks' postpartum. Those women had no exogenous hormonal exposure and avoided any potential negative influence on breast-feeding in the control group, biasing our results toward a larger difference if one had existed. Earlier initiation of hormonal contraception in the immediate postpartum period is a patientcentered strategy to meet women's contraceptive needs.

In many settings, concerns about harmful effects on lactation may currently limit placement of immediate postpartum contraception. This study provides further reassurance that progestin-only contraceptives are unlikely to have a negative impact on breast-feeding. We recommend that immediate postpartum LNG IUD initiation become a standard contraceptive option discussed with pregnant women, regardless of intention to breast-feed.

\section{Acknowledgments}

The research reported in this publication was funded by the Society of Family Planning Research Fund. Use of Research Electronic Data Capture was provided by Eunice Kennedy Shriver National Institute of Child Health and Human Development grant (8UL1TR000105 [formerly UL1RR025764] National Center for Advancing Translational Sciences/National Institutes of Health). Neither entity had any role in research design or execution.

The authors wish to acknowledge Jasmin Alcantara, Eduardo Galindo, and Maria Masters for their efforts in data collection at the University of Utah; Frank Powers, medical providers and staff at the Salt Lake City Community Health Centers; and the residents and nursing staff at both the University of New Mexico and University of Utah Labor and Delivery Units.

\section{References}

1. American Academy of Pediatrics Committee on Breastfeeding. Breastfeeding and the use of human milk. Pediatrics. 2012; 129:e827-41. [PubMed: 22371471]

2. Dykes F, , Moran VH. Infant and young child feeding: challenges to implementing a global strategy Chichester (United Kingdom) and Ames (IA): Wiley-Blackwell; 2009

3. Centers for Disease Control and Prevention. [Accessed Oct. 10, 2016] Infant feeding practices study II and its year six follow up Available at: http://www.cdc.gov/breastfeeding/data/ifps/results.htm

4. National Center for Health StatisticsHealthy People 2020 midcourse review Hyattsville (MD): US Department of Health and Human Services, Centers for Disease Control and Prevention, National Center for Health Statistics; 2016

5. Henderson V, Stumbras K, Caskey R, Haider S, Rankin K, Handler A. Understanding factors associated with postpartum visit attendance and contraception choices: listening to low-income postpartum women and health care providers. Matern Child Health J. 2016; 20:132-43. [PubMed: 27342600]

6. Heller R, Cameron S, Briggs R, Forson N, Glasier A. Postpartum contraception: a missed opportunity to prevent unintended pregnancy and short inter-pregnancy intervals. J Fam Plann Reprod Health Care. 2016; 42:93-8. [PubMed: 26645197]

7. Woo I, Seifert S, Hendricks D, Jamshidi RM, Burke AE, Fox MC. Six-month and 1-year continuation rates following postpartum insertion of implants and intrauterine devices. Contraception. 2015; 92:532-5. [PubMed: 26408376] 
8. Eggebroten JL, Sanders JN, Turok DK. Immediate postpartum intrauterine device and implant program outcomes: a prospective analysis. Am J Obstet Gynecol. 2017; 217:51e1-7. [PubMed: 28342716]

9. Washington CI, Jamshidi R, Thung SF, Nayeri UA, Caughey AB, Werner EF. Timing of postpartum intrauterine device placement: a cost-effectiveness analysis. Fertil Steril. 2015; 103:131-7. [PubMed: 25439838]

10. Neville MC, Morton J. Physiology and endocrine changes underlying human lactogenesis II. J Nutr. 2001; 131:3005S-8S. [PubMed: 11694636]

11. Dunn K, Bayer LL, Mody SK. Postpartum contraception: an exploratory study of lactation consultants' knowledge and practices. Contraception. 2016; 94:87-92. [PubMed: 26996737]

12. Chen BA, Reeves MF, Creinin MD, Schwarz EB. Postplacental or delayed levonorgestrel intrauterine device insertion and breast-feeding duration. Contraception. 2011; 84:499-504. [PubMed: 22018124]

13. Espey E, Ogburn T, Leeman L, Singh R, Ostrom K, Schrader R. Effect of progestin compared with combined oral contraceptive pills on lactation: a randomized controlled trial. Obstet Gynecol. 2012; 119:5-13. [PubMed: 22143258]

14. Gurtcheff SE, Turok DK, Stoddard G, Murphy PA, Gibson M, Jones KP. Lactogenesis after early postpartum use of the contraceptive implant: a randomized controlled trial. Obstet Gynecol. 2011; 117:1114-21. [PubMed: 21508750]

15. Curtis KM, Tepper NK, Jatlaoui TC, et al. US medical eligibility criteria for contraceptive use, 2016. MMWR Recomm Rep. 2016; 65:1-103.

16. Harris PA, Taylor R, Thielke R, Payne J, Gonzalez N, Conde JG. Research Electronic Data Capture (REDCap)-a metadata-driven methodology and workflow process for providing translational research informatics support. J Biomed Inform. 2009; 42:377-81. [PubMed: 18929686]

17. Chapman DJ, Perez-Escamilla R. Maternal perception of the onset of lactation is a valid, public health indicator of lactogenesis stage II. J Nutr. 2000; 130:2972-80. [PubMed: 11110856]

18. Snapinn SM. Noninferiority trials. Curr Control Trials Cardiovasc Med. 2000; 1:19-21. [PubMed: 11714400]

19. Piaggio G, Elbourne DR, Pocock SJ, Evans SJ, Altman DG. CONSORT Group. Reporting of noninferiority and equivalence randomized trials: extension of the CONSORT 2010 statement. JAMA. 2012; 308:2594-604. [PubMed: 23268518]

20. Gabriel AP, Mercado CP. Data retention after a patient withdraws consent in clinical trials. Open Access J Clin Trials. 2011; 3:15-9. [PubMed: 23935390]

21. Phillips SJ, Tepper NK, Kapp N, Nanda K, Temmerman M, Curtis KM. Progestogen-only contraceptive use among breastfeeding women: a systematic review. Contraception. 2016; 94:22652. [PubMed: 26410174]

22. Halderman LD, Nelson AL. Impact of early postpartum administration of progestin-only hormonal contraceptives compared with nonhormonal contraceptives on short-term breast-feeding patterns. Am J Obstet Gynecol. 2002; 186:1250-8. [PubMed: 12066106]

23. Berens P, Labbok M. Academy of Breast-feeding Medicine. ABM clinical protocol no. 13: contraception during breastfeeding, revised 2015. Breastfeed Med. 2015; 10:3-12. [PubMed: 25551519]

24. Brodribb W, Kruske S, Miller YD. Baby-friendly hospital accreditation, in-hospital care practices, and breastfeeding. Pediatrics. 2013; 131:685-92. [PubMed: 23478863]

25. Zhu BP, Rolfs RT, Nangle BE, Horan JM. Effect of the interval between pregnancies on perinatal outcomes. N Engl J Med. 1999; 340:589-94. [PubMed: 10029642]

26. Thiel de Bocanegra H, Chang R, Menz M, Howell M, Darney P. Postpartum contraception in publicly-funded programs and inter-pregnancy intervals. Obstet Gynecol. 2013; 122:296-303. [PubMed: 23969798]

27. Ogburn JA, Espey E, Stonehocker J. Barriers to intrauterine device insertion in postpartum women. Contraception. 2005; 72:426-9. [PubMed: 16307964] 


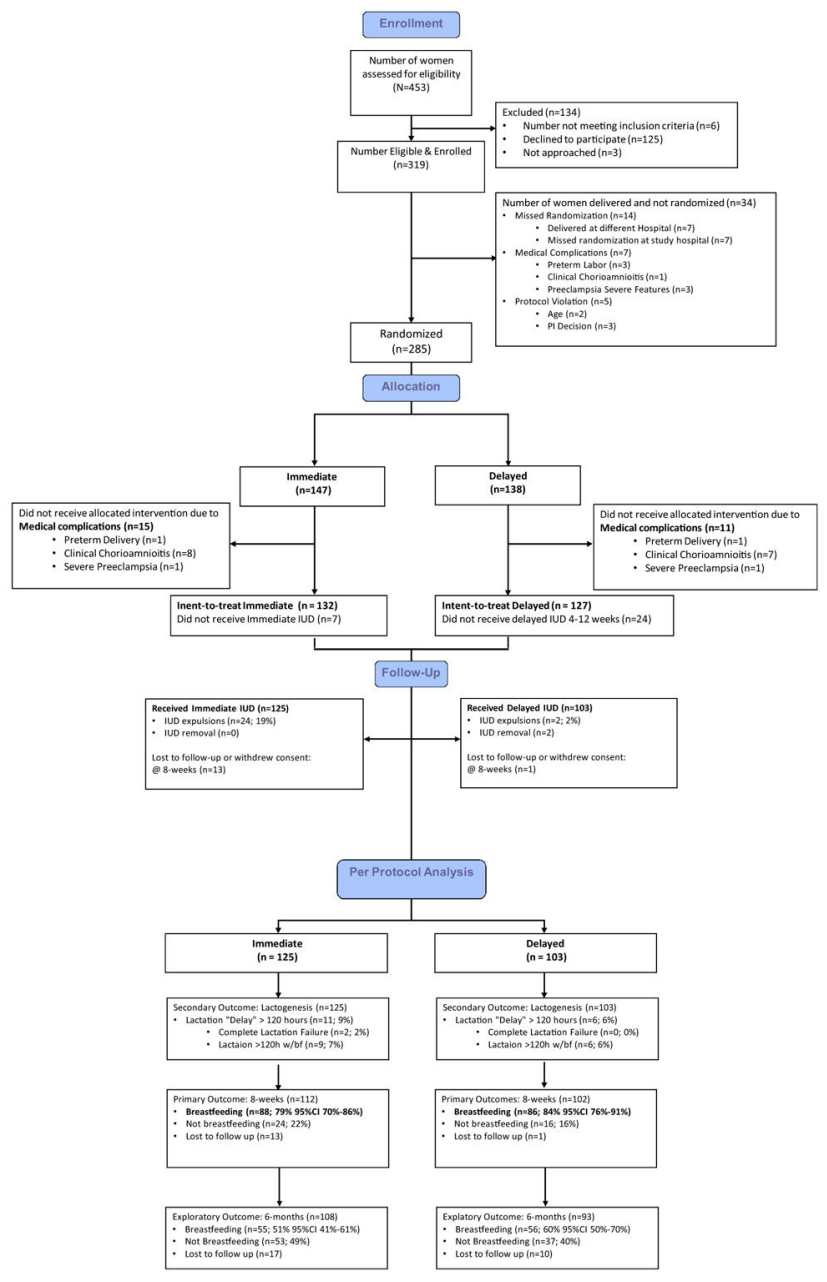

FIGURE 1. Consolidated Standards of Reporting Trials (CONSORT) flow chart

Noninferiority CONSORT diagram. Breakdown in participant enrollment and allocation, including follow-up for primary outcome at 8 weeks and final outcome at 6 months. 


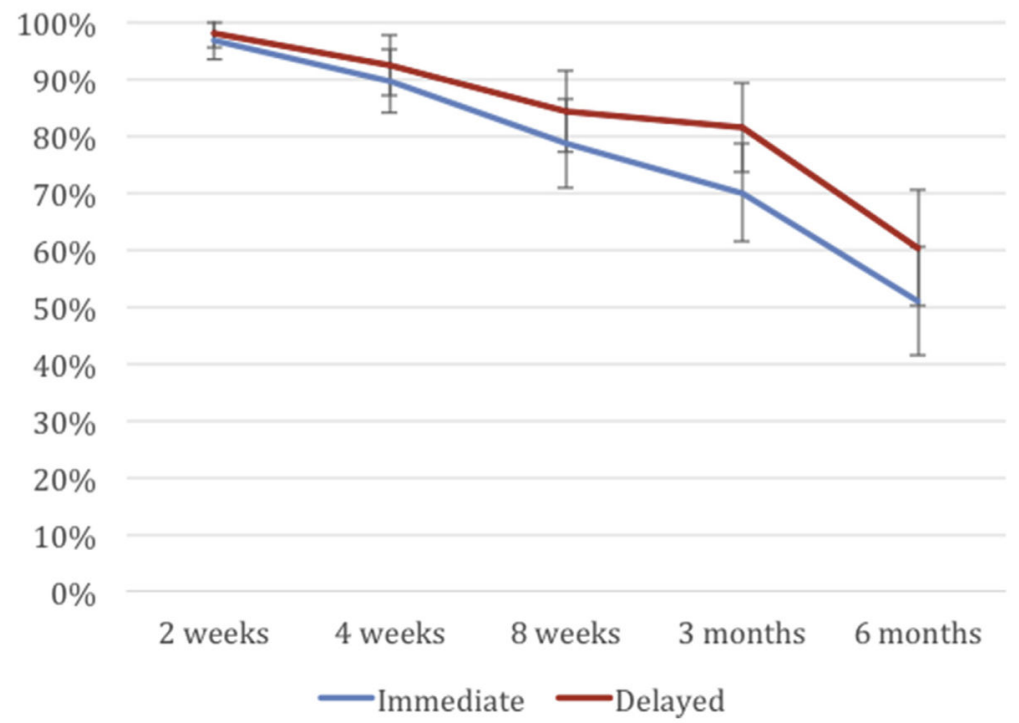

FIGURE 2. Any breast-feeding reported from 2 weeks to 6 months postpartum

Curve demonstrates noninferiority for primary outcome of any breast-feeding at 8 weeks as point estimate for 8 -week breast-feeding continuation for early group falls within $15 \%$ of upper end of $95 \%$ confidence interval for late insertion group. 


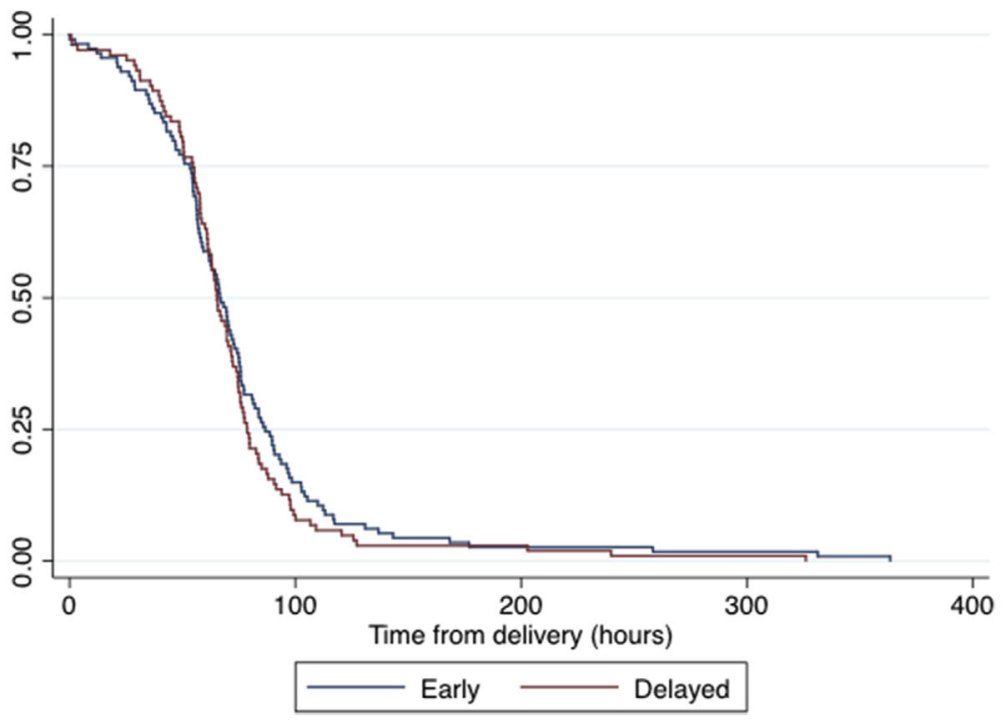

FIGURE 3. Kaplan-Meier survival curve of time to lactogenesis

Noninferiority for secondary outcome of time to lactogenesis. 
$100 \%$

$90 \%$

$80 \%$

$70 \%$

$60 \%$

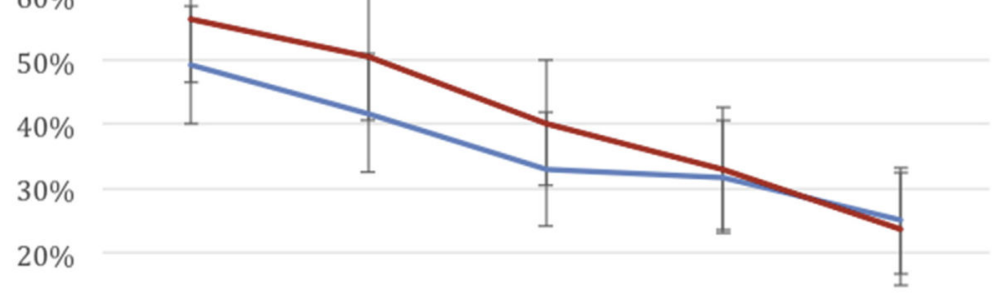

$10 \%$

$0 \%$

$$
\begin{array}{ll}
2 \text { weeks } & 4 \text { weeks } \quad 8 \text { weeks } \quad 3 \text { months } 6 \text { months } \\
& \text { - Immediate }- \text { Delayed }
\end{array}
$$

FIGURE 4. Report of exclusive breast-feeding out to 6 months

Exclusive breast-feeding between groups differed slightly at 8 weeks but this resolved by 6 months. 


\section{TABLE}

Demographics by randomization (intent-to-treat)

\begin{tabular}{|c|c|c|}
\hline & $\begin{array}{c}\text { Immediate, n (\%) } \\
132(51)\end{array}$ & $\begin{array}{c}\text { Delayed, n (\%) } \\
127(49)\end{array}$ \\
\hline \multicolumn{3}{|l|}{ Age at delivery, y } \\
\hline$<19$ & $5(4)$ & $7(6)$ \\
\hline $20-24$ & $30(23)$ & $32(26)$ \\
\hline $25-29$ & $39(30)$ & $33(27)$ \\
\hline $30-34$ & $37(28)$ & $35(29)$ \\
\hline $35-39$ & $18(14)$ & $14(11)$ \\
\hline $40-44$ & $2(2)$ & $2(2)$ \\
\hline \multicolumn{3}{|l|}{ Parity } \\
\hline 1 & $58(44)$ & $55(43)$ \\
\hline 2 & $24(18)$ & $21(17)$ \\
\hline$\geq 3$ & $50(38)$ & $51(40)$ \\
\hline \multicolumn{3}{|l|}{ Race } \\
\hline American Indian or Alaska Native & $3(2)$ & $7(6)$ \\
\hline Asian & $1(1)$ & $1(1)$ \\
\hline Native Hawaiian/Pacific Islander & $0(0)$ & $1(1)$ \\
\hline White & $104(79)$ & $92(73)$ \\
\hline Other & $20(15)$ & $22(18)$ \\
\hline \multicolumn{3}{|l|}{ Ethnicity } \\
\hline Hispanic or Latino & $106(80)$ & $106(84)$ \\
\hline Non-Hispanic & $26(20)$ & $21(17)$ \\
\hline \multicolumn{3}{|l|}{ Epidural } \\
\hline Yes & $87(67)$ & $87(71)$ \\
\hline No & $43(33)$ & $36(29)$ \\
\hline \multicolumn{3}{|c|}{ For how long are you planning to breast-feed your baby? } \\
\hline$<6$ mo & $17(13)$ & $17(14)$ \\
\hline$>6 \mathrm{mo}$ & $113(87)$ & $107(86)$ \\
\hline \multicolumn{3}{|l|}{ Prior breast-feeding experience? } \\
\hline Yes & $99(76)$ & $88(71)$ \\
\hline No & $32(24)$ & $36(29)$ \\
\hline \multicolumn{3}{|l|}{ Mode of delivery } \\
\hline Spontaneous vaginal & $108(83)$ & $101(82)$ \\
\hline Forceps/vacuum & $1(1)$ & $8(7)$ \\
\hline Cesarean & $21(16)$ & $15(12)$ \\
\hline \multicolumn{3}{|l|}{ Recruitment site } \\
\hline University of Utah & $73(55)$ & $76(60)$ \\
\hline University of New Mexico & $59(45)$ & $51(40)$ \\
\hline
\end{tabular}


Turok et al. Breastfeeding Levonorgestrel IUD Study. Am J Obstet Gynecol 2017. 